\title{
COMPENSACIÓN DE LOS EFECTOS DISPERSIVOS DE LA FIBRA ÓPTICA EN UN SISTEMA IM-DD USANDO ECUALIZACIÓN LMS Y MLSE
}

\author{
OPTICAL FIBER IMPAIRMENTS COMPENSATION IN A IM-DD \\ SYSTEM USING LMS AND MLSE EQUALIZATION
}

Roobert David Gallego Ruiz'르, Yeison David Sánchez Sierra ${ }^{1}$, Jhon James Granada Torres ${ }^{1}$, Ana María Cárdenas Soto ${ }^{1}$, Neil Guerrero González ${ }^{2}$

1. Universidad de Antioquia, Colombia.

2. Tyndall National Institute. Cork, Ireland.

Recibido: 3 Diciembre Aceptado: 15 Diciembre

*Correspondencia del autor. Calle 70 No. 52-21, Of. 18-310 Medellín, Colombia. E-mail: jhon.granada@udea.edu.co

\section{RESUMEN}

Actualmente en Colombia ha comenzado el despliegue de la fibra óptica hasta el hogar, con el fin de que los operadores de telecomunicaciones ofrezcan servicios de mayor capacidad, aprovechando el gran ancho de banda que proporciona la fibra óptica. Para la masificación de esta tecnología y el cubrimiento de todos los usuarios finales, las redes deben ser de bajo costo, por lo cual los sistemas modulados en intensidad con detección directa (IM-DD) siguen vigentes a pesar de las ventajas de los receptores ópticos coherentes. No obstante, en los sistemas IM-DD, las señales transmitidas se ven más afectadas ante las imperfecciones de la fibra óptica como lo es la dispersión cromática, causante de la interferencia inter-símbolo, la cual provoca el aumento de la tasa de error de bit (BER). En este artículo se presenta una red óptica IM-DD simulada en Virtual Photonics ${ }^{\circledR}(\mathrm{VPI})$, para transmisión de señales con modulación No-Return-to-Zero On-Off-Keying (NRZ-OOK) de hasta $40 \mathrm{Gbps}$, con longitudes de fibra óptica monomodo estándar hasta de $80 \mathrm{~km}$. Se plantea la compensación de los efectos del canal óptico mediante la ecualización eléctrica usando el algoritmo de LMS (Least-Mean-Square), estimando los coeficientes del canal a través de secuencias de entrenamiento, presentando una reducción significativa del BER a 10 Gbps con respecto a la relación señal a ruido (SNR). Además, se comparan los resultados con un ecualizador MLSE (Maximunlikelihood sequence estimation), el cual reduce en menor medida el BER en comparación a la ecualización LMS..

Palabras claves: Ecualización, Fibra óptica, LMS, MLSE, Tasa de Error de bit (BER). 


\begin{abstract}
Currently, Colombia has started the deployment of fiber-to-the-home (FTTH) technology. It is due to the traffic demand and the new services offered to the end users, taking advantage of the great optical fiber bandwidth. For FTTH massification, the equipments of optical networks must be of low cost, therefore, IM-DD (intensity modulation with direct detection) systems are still implemented in spite of the advantages of coherent detection systems. However, in IM-DD systems, the transmitted signals are not tolerable to the optical fiber impairments, mainly, the chromatic dispersion, which generates the inter-symbol interference (ISI) in the received signal, increasing the bit error rate (BER). In this paper, an IM-DD system is modeled in Virtual Photonics $($ VPI) software. The optical network is modulated in No-Return-to-Zero On-Off-Keying (NRZ-OOK) format, at 40 Gbps, reaching distances of $80 \mathrm{~km}$. Optical fiber impairments compensation is proposed using LMS (LeastMean-Square) algorithm, which uses a training sequence to find the channel coefficients, and MLSE (Maximunlikelihood sequence estimation). These techniques allow a BER reduction and improve the system performance.
\end{abstract}

Keywords: Equalization, Optical Fiber, Least-Mean-Square (LMS), Maximun-likelihood sequence estimation (MLSE), Bit-error-rate (BER).

\section{1-INTRODUCCIÓN}

El avance en los sistemas de telecomunicaciones de alta capacidad se ha dado gracias a las transmisiones por fibra óptica en conjunto con el desarrollo de transmisores y receptores que operan a una tasa del orden de los Gbps. Pasando de una modulación directa del láser a usar moduladores externos de intensidad y fase, como el Mach-Zender (MZM) y el modulador de electro-absorción (EAM)(1). Los sistemas ópticos con modulación de Intensidad y Detección Directa (IM-DD) son ampliamente usados debido a que son de bajo costo y de fácil implementación(2), comparado con la tecnología de detección coherente, que permite mayor capacidad de transmisión(3), pero a cambio de un costo muy elevado para el despliegue actual en los terminales de usuario final(2).

La tecnología de fibra hasta el hogar (FTTH, fiber tothe-home) se ha desplegado en diferentes países, con la intención de ofrecer más capacidad a los usuarios finales en comparación con las tecnologías de cobre, evitando el cuello de botella generado en las interfaces que conectan a los medios de cobre como el cable coaxial(4). Las transmisiones se ven restringidas en distancia y velocidad de transmisión, debido a las imperfecciones propias de la fibra óptica, como la dispersión cromática (CD), la dispersión por modo de polarización (PMD), ruidos térmicos y ruidos generados por los dispositivos electro-ópticos, los cuales generan interferencia intersímbolo (ISI), cuyo efecto in- fluye en el aumento de la tasa de error de bit (BER)5. La ISI puede ser compensada mediante diferentes técnicas ópticas y eléctricas que permiten reducir el BER. La dispersión cromática puede ser mitigada de manera pasiva al instalar fibras compensadoras de dispersión (DCF) cuya principal característica es su elevado parámetro de dispersión y de signo opuesto al de las fibras convencionales operando en tercera ventana6. Las técnicas eléctricas comúnmente usadas son los ecualizadores eléctrico, que por medio de algoritmos que modifican los coeficientes de filtros adaptativos de acuerdo al comportamiento de la señal recibida que se compara con una secuencia de entrenamiento o con estimaciones probabilísticas (ecualización ciega) (7).

Entre los ecualizadores más estudiados se encuentran los transversales como el ZF (Zero-Forcing) y el MMSE (Minimum Mean Square error), los ecualizadores fraccionados y otros de mayor complejidad como el DFE (Decision Feedback Equalizer), con estimación probabilística como el MLSE (Maximum likelihood sequence estimation) y de secuencias de entrenamiento como el LMS (Leans Means Square) $(8,9,10)$.

El algoritmo adaptativo LMS se caracteriza por su fácil implementación, bajo gasto computacional y su efectividad en la modificación de los coeficientes adaptativos del ecualizador, minimizando las 
imperfecciones que se presentan en la señal transmitida por la fibra(11); realiza la corrección de los desplazamientos aleatorios en la frecuencia de los sistemas QAM de orden superior(12), y en las nuevas modulaciones sin portadora tipo CAP (Carrierless amplitude/phase), a tasas de transmisión hasta de $40 \mathrm{Gbps}$, en $40 \mathrm{Km}$ de fibra estándar monomodo(13). Además, se han mostrado resultados experimentales donde la ecualización LMS ha mitigado efectos no lineales en un sistema óptico multiportadora a velocidades en el rango de los Gbps(13).

Además del LMS, el algoritmo MLSE también es usado en aplicaciones ópticas. Este algoritmo realiza el cálculo del error mínimo aproximado en la señal transmitida mediante el algoritmo de Viterbi (VA), una técnica eficiente en el cálculo probabilístico de detección de señales afectadas por la $\mathrm{CD}$ y el ruido(14).

Los algoritmos LMS y MLSE continúan en constante investigación, optimizándolos en términos de eficiencia computacional para los futuros sistemas ópticos de gran capacidad(15). Entre los algoritmos LMS modificados se encuentran el M-LMS7 y el DDLMS(13) que alcanzan una mayor exactitud y una rápida convergencia.

En la sección II se presentará una red óptica IM-DD simulada en Virtual Photonics ${ }^{\circledR}(\mathrm{VPI})$, para transmisiones del orden de los Gbps, con longitudes de fibra óptica monomodo estándar de hasta $80 \mathrm{~km}$, donde se compensan los efectos del canal óptico mediante la ecualización eléctrica usando el algoritmo LMS y MLSE. Se compara el rendimiento del sistema ecualizado mediante la medida de la magnitud vectorial de las señales muestreadas y las curvas de tasa de error de bit (BER) versus relación señal a ruido (SNR).

\section{2- MONTAJE DEL SISTEMA DE TRANSMI- SIÓN}

Las simulaciones de las transmisiones de la red óptica IM-DD desarrolladas en el software Virtual Photonics Inc ${ }^{\circledR}$ (VPI), para transmisión de señales con modulación en amplitud permiten evaluar el impacto de los efectos no deseados en las señales demoduladas. A continuación se muestra la configuración utilizada:

\section{OFICINA CENTRAL}

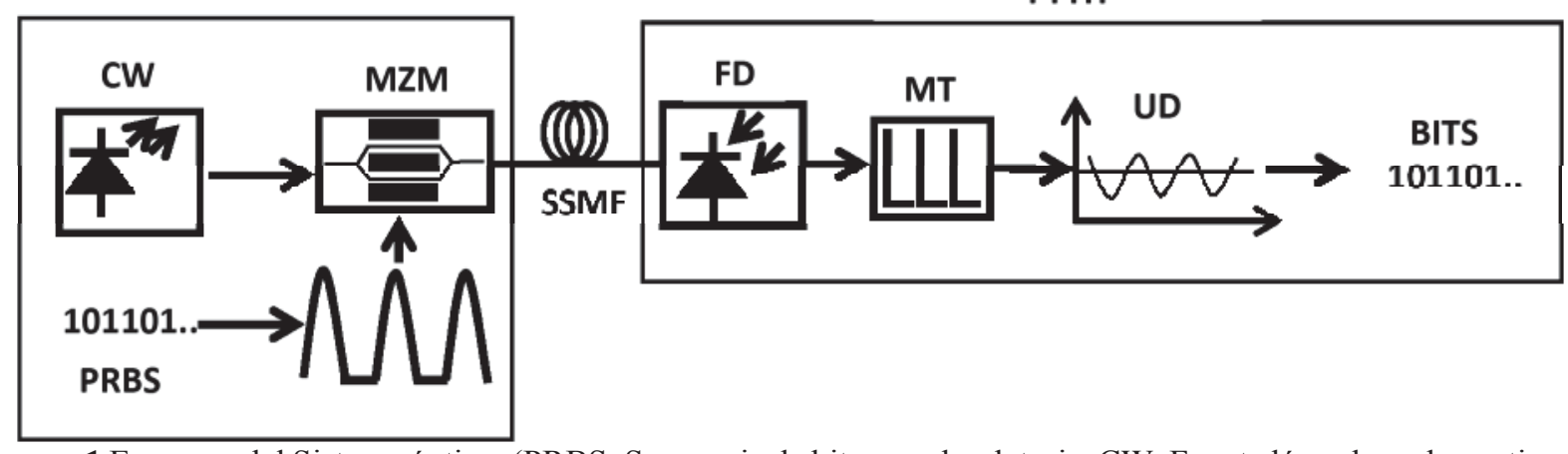

Figura. 1 Esquema del Sistema óptico. (PRBS: Secuencia de bits pseudo-aletoria, CW: Fuente láser de onda continua, FP. Formación de pulsos, FD: Foto detector, ME: Modulador Externo SSMF: Fibra monomodo estándar, MT: Muestreador, UD: Umbral de decisión)

El transmisor consiste en una fuente laser de onda continua con un ancho de línea de $1 \mathrm{MHz}$ en el rango de $1550 \mathrm{~nm}$. La potencia óptica del láser es $1 \mathrm{dBm}$. La salida del láser está conectada a un modulador de intensidad externo con características ideales. Las secuencias de bits se encuentran moduladas digitalmente en el formato eléctrico NRZ-OOK a 10 y 40 Gbps.

El canal de transmisión es una fibra monomodo estándar con una longitud de hasta $80 \mathrm{Km}$, con una atenuación de $0.2 \mathrm{~dB} / \mathrm{Km}$ y un factor de dispersión de $16 \mathrm{ps} /$ nm.km. En el receptor las señales transmitidas son de- tectadas por un fotodiodo de responsividad de $1 \mathrm{~A} / \mathrm{W}$. La señal eléctrica NRZ-OOK es muestreada, y por medio del umbral de decisión se obtiene la secuencia de bits.

\section{ECUALIZACIÓN DEL CANAL}

Se desea compensar los efectos del canal por medio de la ecualización adaptativa LMS y MLSE en el dominio eléctrico.

\section{A- Modelo del canal}

En ausencia de no linealidades, el efecto de la disper- 
sión cromática, sobre la envolvente $\mathrm{A}(\mathrm{z}, \mathrm{t})$ de un pulso se puede representar con la ecuación parcial diferencial (1), siendo el factor más influyente en la fibra óptica para modulación NRZ-OOK.

$$
\frac{2 A(z, t)}{2 z}=j \frac{D \lambda}{4 \pi c} \frac{2 A(z, t)}{2 t^{2}}
$$

Donde D es el coeficiente de dispersión de la fibra, z es la propagación, $t$ es la variable del tiempo en una trama de movimiento del pulso, c es la velocidad de la luz $\lambda$ es la longitud de onda(16).

\section{B- Ecualización LMS}

El LMS es un algoritmo iterativo que encuentra los coeficientes de un filtro adaptativo, mediante la minimización del error generado entre la diferencia de la señal transmitida y la señal recibida(16,17), definido por las siguientes expresiones:

$$
\begin{aligned}
& y(n)=\boldsymbol{w}^{T}(n-1) \boldsymbol{u}(n) \\
& \mathrm{e}(n)=d(n)-y(n) \\
& \boldsymbol{w}(n)=\boldsymbol{w}(n-1)+f(\boldsymbol{u}(n), e(n), \mu)
\end{aligned}
$$

La ecualización de actualización de pesos está dada por:

$$
f(u(n), e(n), \mu)=\mu e(n) u^{*}(n)
$$

Dónde:

$n$ : Posición actual en el tiempo.

$w(n)$ : Vector de los coeficientes del filtro en el tiempo.

$d(n)$ : Secuencia de entrenamiento (señal deseada).

$y(n)$ : Señal a la salida del ecualizador.

$u(n)$ : Señal recibida, que ingresa al ecualizador.

$u^{*}(n)$ : Conjugado de $u(n)$.

$e$ : Error estimado en el tiempo.

El LMS realiza un proceso iterativo según los parámetros de tamaño del filtro adaptativo y tamaño de paso. En la figura 2 se muestra la configuración del receptor. Se usó un filtro de orden 4 con un tamaño de paso de 0.06 .

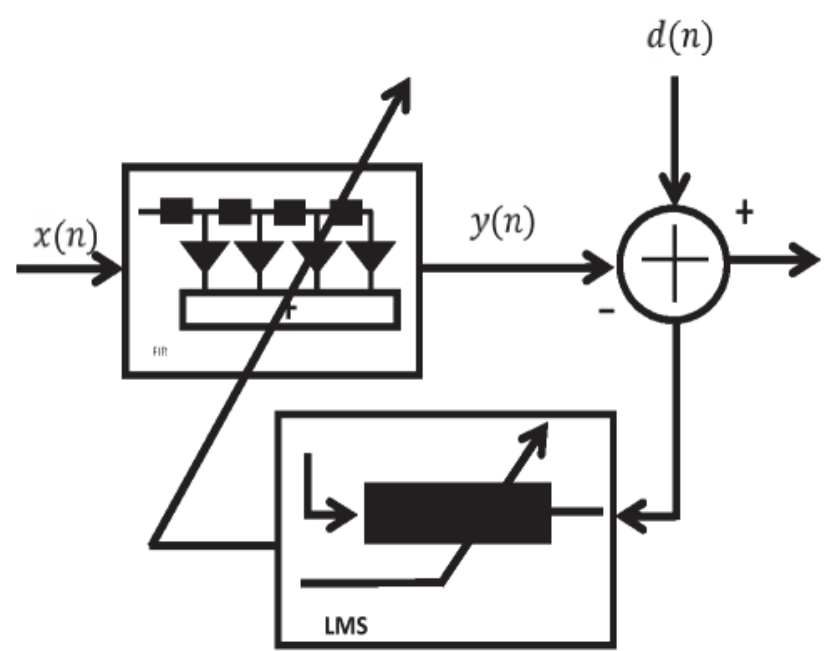

Figura. 2 Ecualización con LMS (MT: Muestreador, UD: Umbral de decisión)

\section{C- Ecualización MLSE}

El MLSE minimiza la probabilidad de error en la toma de las decisiones sobre la secuencia transmitida, detectando óptimamente la secuencia de entrenamiento18-19. El receptor emula el canal distorsionado a través de los coeficientes estimados (Ver fig. 3) y calcula probabilísticamente los bits transmitidos, de acuerdo a los valores recibidos de la señal digitalizada.

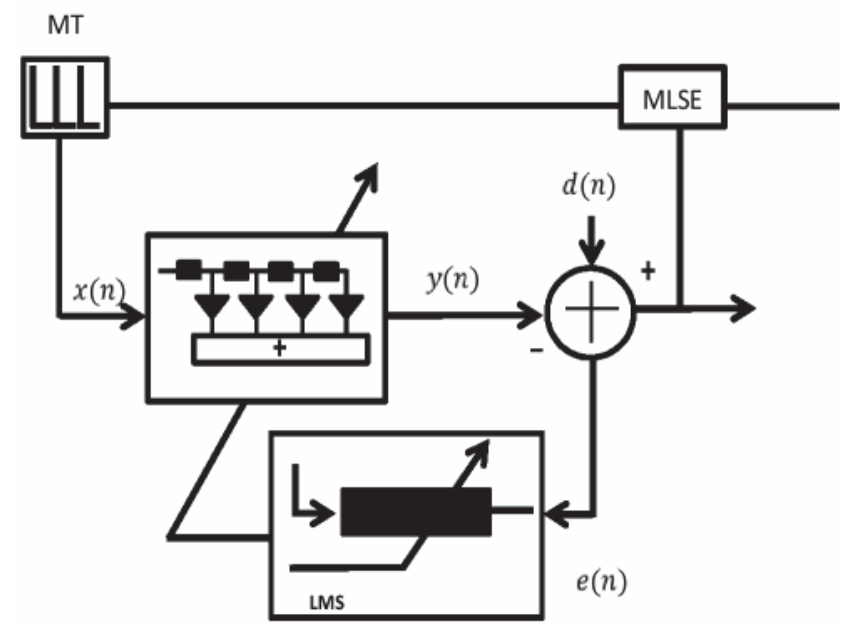

Figura. 3 Ecualización con MLSE

La base del método MLSE es el valor del parámetro que maximiza el valor de la función de probabilidad y esto se aplica a un sistema de transmisión, al suponerse que se recibe el vector de una señal $r=\left[\begin{array}{llll}r_{0} & , r_{1} & , \ldots & , r_{(N-1)}\end{array}\right]$ considerando que representa una de las posibles secuencias transmitidas $\left[S_{0}, S_{1}, \ldots, S_{(N-1)}\right]$ por lo tanto la secuencia más probable es:

$$
s(r)=\arg _{\mathrm{st}} \max f\left(r \mid s_{\mathrm{t}}\right)
$$


La secuencia más probable para ser transmitida es la asociada con la mínima distancia euclidiana entre la señal recibida y la posible secuencia trasmitida $S t$ :

$$
\Lambda=/_{k=0}^{N-1} \mathbf{m}\left(x_{k}, x_{k+1}\right)
$$

Estas consideraciones sugieren una formula recursiva de:

$$
\Lambda\left(s_{t}\right) \Lambda\left(s_{k}\right)=\Lambda\left(s_{k+1}\right)+\lambda\left(x_{k}, x_{k+1}\right)
$$

Con $S_{i}=\left[S_{0}, S_{1}, \ldots, S_{N-1}\right]\left(S_{N-1}=S_{t}\right)$ y $\Lambda\left(s_{0}\right)=0$ Donde después de iteraciones se obtiene:

$$
\Lambda\left(\mathrm{s}_{\mathrm{t}}\right)=\Lambda\left(s_{N-1}\right)
$$

\section{RESULTADOS Y DISCUSIONES}

La señal muestreada en el receptor, se muestra como una constelación con los puntos dispersos, cercanos al ' 0 ' y ' 1 ' lógico como se muestra en la figura 5. Se calcula el valor EVM (Error Vector Magnitude) definida como la raíz cuadrada media (RMS) entre un conjunto de símbolos medidos y símbolos ideales(20). En una modulación de amplitud se puede determinar de la siguiente manera:

$$
E V M_{R M S}=\left(\frac{\frac{1}{T} \sum_{t=1}^{T}\left|I_{t}-\boldsymbol{I}_{o, t}\right|^{2}}{\frac{1}{N} \sum_{n=1}^{N}\left[\left(I_{o, n}\right)^{2}\right]}\right)^{\frac{1}{2}}
$$

Dónde:

$T$ : Número de símbolos de la constelación medida.

$N$ : Número de símbolos de la constelación ideal.

$I_{t}$ : Voltaje normalizado de los símbolos recibidos.

$I_{0, t}:$ Voltaje normalizado para los símbolos ideales transmitidos.

(a)

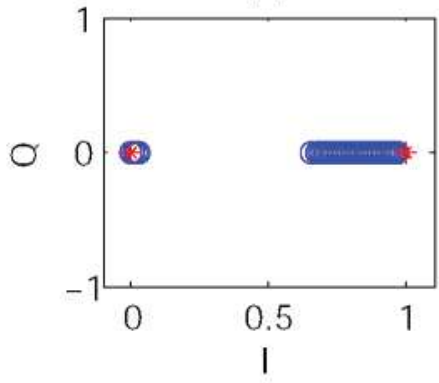

(b)

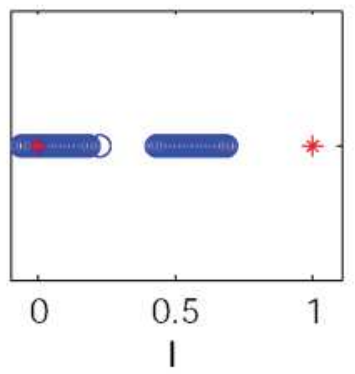

La figura 5a se muestra las curvas BER vs SNR para una transmisión a $10 \mathrm{Gbps}$. Aquí se compara la transmisión ecualizada y sin ecualizar. Usando ecualización LMS se logró una reducción del $\log (\mathrm{BER})$ de -0.5 a -1.7 con SNR de $1 \mathrm{~dB}$. Además, se presenta una disminución del SNR aproximada de $16 \mathrm{~dB}$ a $3 \mathrm{~dB}$ para un $\log (\mathrm{BER})$ de -2.1 a una distancia de $5 \mathrm{Km}$, mientras que para $80 \mathrm{Km}$ se observó una disminución del $\log (\mathrm{BER})$ de $\sim-0.4 \mathrm{a} \sim-1$ con una SNR de $1 \mathrm{~dB}$ y una penalidad SNR de $\sim 21$ a $\sim 15$ con un $\log$ (BER) de -2.1. Lo anterior muestra que el LMS es más eficiente a distancias menores de $20 \mathrm{Km}$.

Los diagramas de constelación de la Figura 5 para una transmisión a 5 y a $80 \mathrm{~km}$. A $80 \mathrm{~km}$ (fig. 5c), se miden un EVM de $44.31 \%$ sin ecualizar. Con ecualización LMS (fig. 5e) el EVM es del 30.90\%, mostrando una reducción en la dispersión de la señal muestreada. Para el caso de $5 \mathrm{~km}$, se midió un valor de EVM de $14.31 \%$ sin ecualizar (fig. 5b) y un $7.79 \%$ con LMS (fig. 5d), reduciendo aproximadamente a la mitad el valor porcentual de EVM.

En la figura 6 se muestran las curvas BER vs SNR comparando la señal sin ecualizar y con ecualización MLSE. Se puede notar que la ecualización MLSE muestra una menor reducción de $\log (\mathrm{BER})$ y de la penalidad de la SNR en comparación con el LMS. A una distancia de $5 \mathrm{~km}$ se observa una reducción aproximada del $\log ($ BER ) de -1.1 a -1.6 con una SNR de $13 \mathrm{~dB}$. Además, se muestra una reducción en la penalidad de la SNR de $18 \mathrm{~dB}$ a $14 \mathrm{~dB}$ para un $\log (\mathrm{BER})$ de -1.8 a una distancia de $5 \mathrm{~km}$. Para una transmisión de 20 $\mathrm{Km}$ se visualiza para una SNR de $13 \mathrm{~dB}$ una reducción aproximada del log BER de -1.2 a -1.4, y una disminución en la penalidad de la SNR de $18 \mathrm{~dB}$ a $16 \mathrm{~dB}$ para un $\log (\mathrm{BER})$ de -1.8

Figura. 4 Diagrama de constelación, sin ecualización. (a) Señal transmitida a 10 Gbps a una distancia de $20 \mathrm{Km}$ (b) Señal transmitida a $10 \mathrm{Gbps}$ a una distancia de $80 \mathrm{Km}$ (c) Señal transmitida a 40 Gbps a una distancia de 20 Km (d) Señal transmitida a 40 Gbps a una distancia de $80 \mathrm{Km}$. 

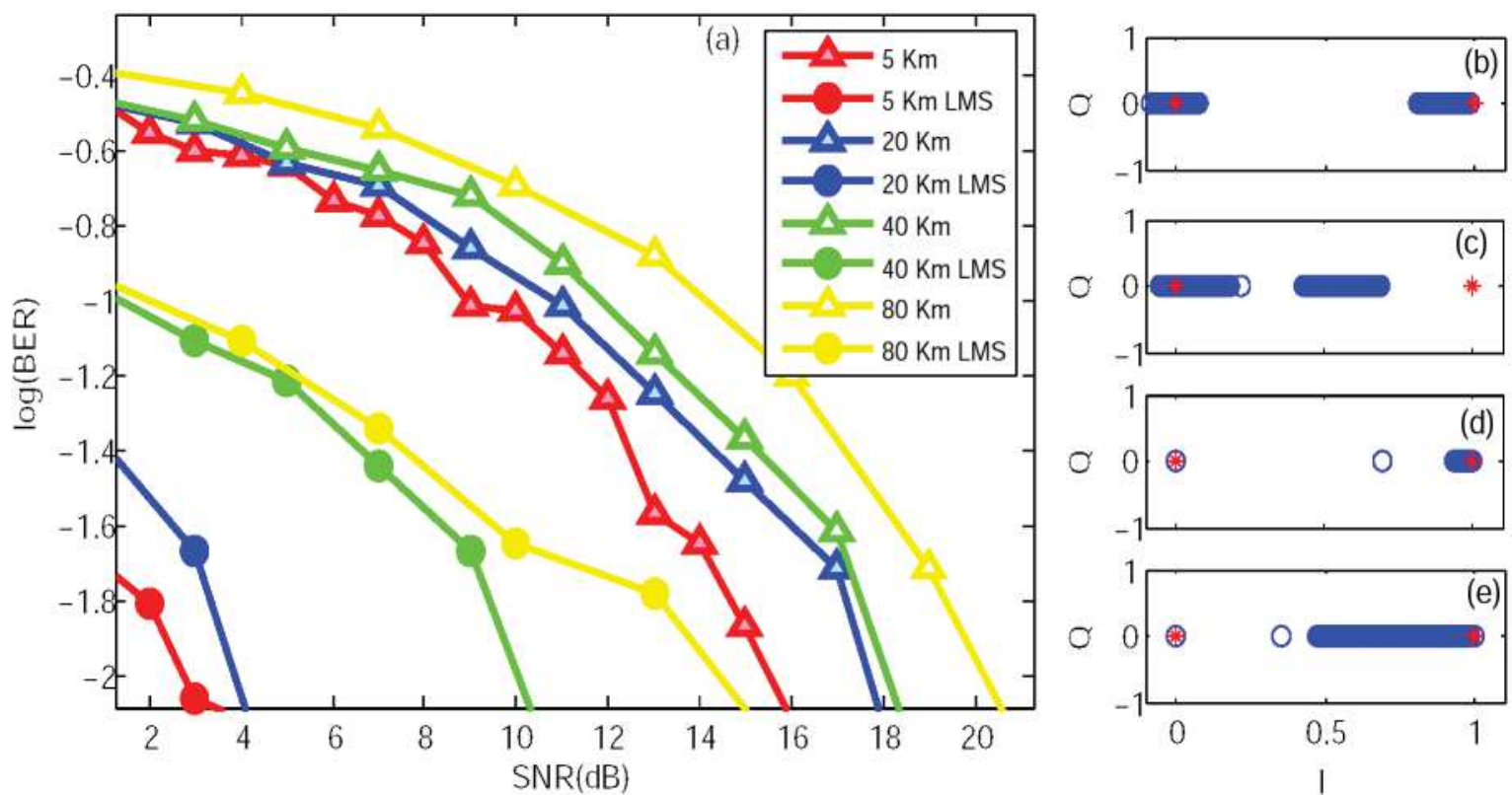

Figura.5 (a) Curvas BER vs SNR para una transmisión a 10 Gbps, con y sin ecualización. Diagrama de constelación para una transmisión a $10 \mathrm{Gbps}$ (b) a una distancia de $5 \mathrm{Km}$ sin ecualización (c) a una distancia de $80 \mathrm{Km}$ sin ecualización (d) a una distancia de $5 \mathrm{Km}$ con ecualización (e) a una distancia de $80 \mathrm{Km}$ con ecualización.

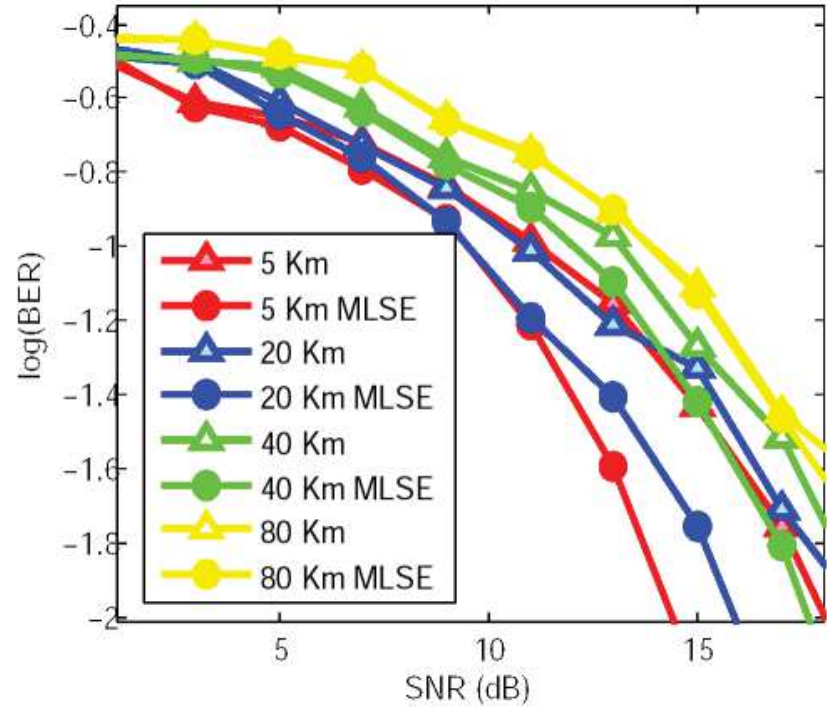

Figura. 6 Curvas BER vs SNR para una transmisión a 10 Gbps, con y sin ecualización.

La Figura 7a muestra las curvas BER vs SNR para transmisiones a 40 Gbps con y sin ecualización LMS. A una distancia de $5 \mathrm{Km}$ el $\log (\mathrm{BER})$ se reduce de $\sim-0.4 \mathrm{a} \sim-1.5$ con un SNR de $1 \mathrm{~dB}$ gracias a la ecualización LMS. Así mismo, se reduce la penalidad del SNR en $17 \mathrm{~dB}$ con un $\log (\mathrm{BER}) \mathrm{de}-2$.
En los diagramas de constelación de la figura 7 para la transmisión de 40 Gbps. Se obtiene un EVM de $46.36 \%$ sin ecualizar (fig. 7 b) y un $12.79 \%$ ecualizado para distancia de $5 \mathrm{Km}$ (fig. 7c). Para distancia de 80 $\mathrm{km}$ el EVM tiene un valor de $85.07 \%$ sin ecualizar y de $60.91 \%$ con ecualización LMS. Lo anterior muestra que para distancia de $5 \mathrm{Km}$, el LMS disminuye en más de una tercera parte su valor porcentual de EVM. Para una distancia de $80 \mathrm{Km}$ el valor porcentual EVM es superior al 50\% incluso con ecualización.

En la Figura 8 se muestran las curvas BER vs SNR para una transmisión a 40 Gbps con y sin ecualización MLSE. Las curvas sin ecualización muestran una transmisión con $\log (\mathrm{BER})$ mayor a -0.6 , lo que explica una transmisión de información no recuperable. Con ecualización MLSE hubo una disminución de aproximadamente $1 \mathrm{~dB}$ en la penalidad de la SNR para $5 \mathrm{~km}$. Sin embargo, el comportamiento es muy similar entre la señal ecualizada y sin ecualizar, lo que demuestra que la ecualización MLSE no es eficiente a esta velocidad de transmisión debido a que no hubo una reducción significativa de la BER. 

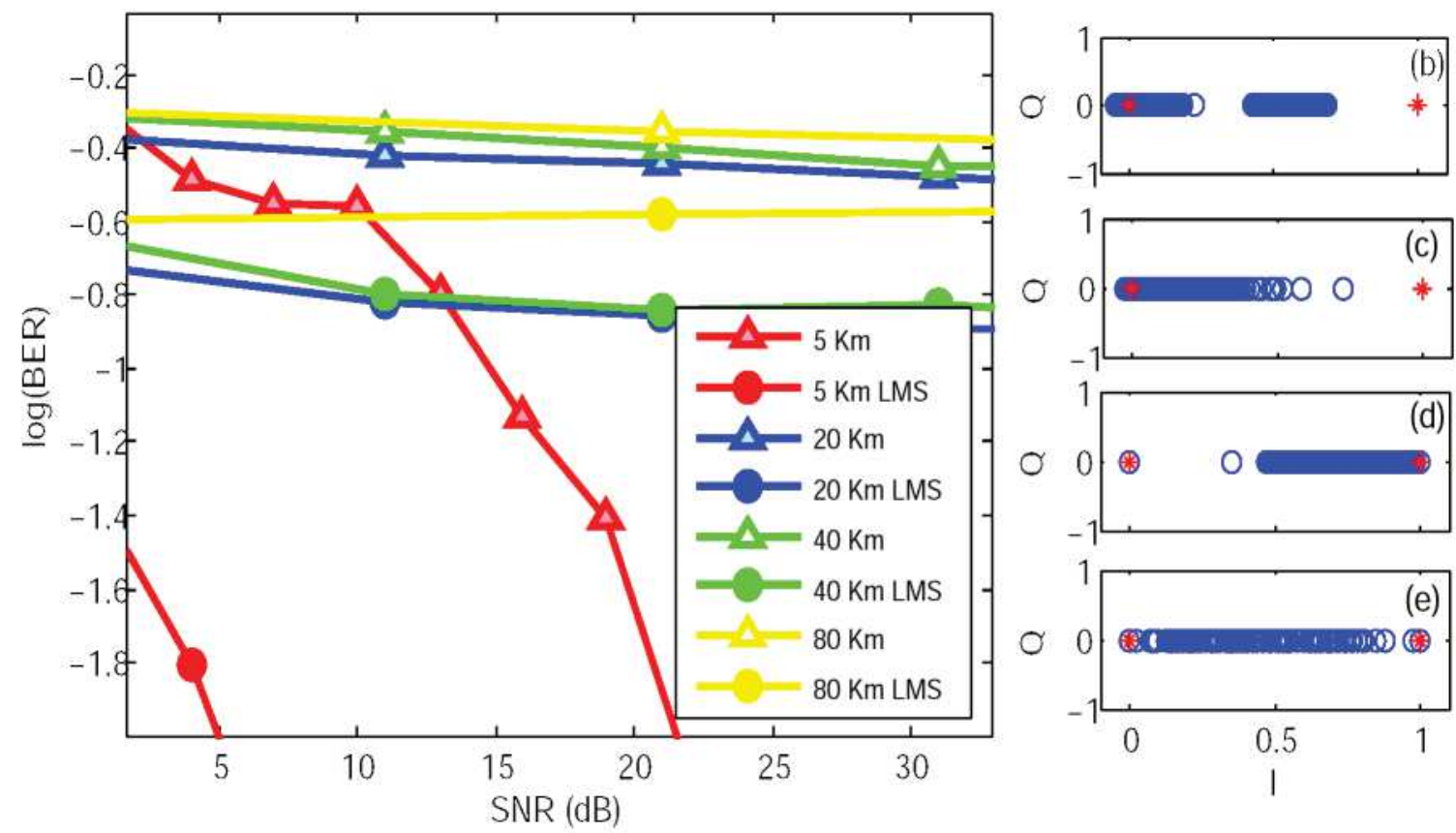

Figura 7 (a) Curvas BER vs SNR para una transmisión a 40 Gbps, con y sin ecualización. Diagrama de constelación para una transmisión a $40 \mathrm{Gbps}$ (b) a una distancia de $5 \mathrm{Km}$ sin ecualización (c) a una distancia de $80 \mathrm{Km}$ sin ecualización (d) a una distancia de $5 \mathrm{Km}$ con ecualización (e) a una distancia de $80 \mathrm{Km}$ con ecualización

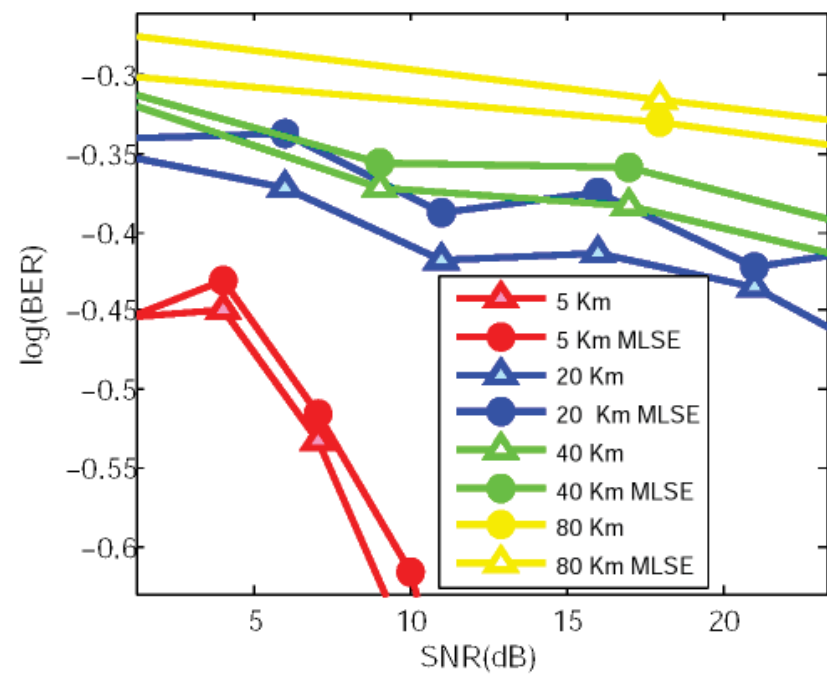

Figura. 8 Curvas BER vs SNR para una transmisión a 40 Gbps, con y sin ecualización.

\section{CONCLUSIONES}

Se analizó un sistema IM-DD con modulación NRZOOK hasta los $40 \mathrm{Gbps}$. A esta tasa de transmisión la información de la señal no puede ser recuperada sin usar métodos de ecualización. Con el algoritmo de LMS, se pudo lograr una disminución significativa en la penalidad de la SNR para distancias menores a los $5 \mathrm{~km}$. Además, la ecualización LMS reduce de manera eficiente el BER a velocidades de $10 \mathrm{Gbps}$ hasta los $80 \mathrm{~km}$. El algoritmo de MLSE no fue eficiente en la reducción de la BER para los casos transmitidos, mostrando sólo resultados relevantes a transmisión de $10 \mathrm{Gbps}$ con distancias menores a los $20 \mathrm{~km}$. Además, el gasto computacional del algoritmo MLSE es mayor que el del LMS. 


\section{BIBLIOGRAFÍA}

1. Deming K, Hui W, Yan L, Jizhao Z, Siyuan Z, Xixue J, et al. Ultra-low timing-jitter 40 Ghz clock recovery using EAM-MZM double-loop and its application in a $640 \mathrm{Gbit} / \mathrm{s}$ OTDM system. In Optical Communications (ECOC), 2012 38th European conference exhibition; 2012.

2. España Bosquera M. Fotodetectores y receptores para comunicaciones ópticas. J Bravo, Ediciones Díaz de Santos S.A. 2005.

3. Alzate D, Cardenas A. Retos en la transmisión de 40/100 Gb/s sobre fibra óptica. Revista en telecomunicaciones e informatica. 2011; 1(2).

4. Tinoco J. Estudio y diseño de una red de fibra óptica FTTH para brindar servicio de voz, video y datos para la urbanizacion los olivos ubicada en el sector toctesol en la parroquia borrero de la ciudad de azogues. 2011. Tesis de Grado, Dep. Ingeníeria Electrónica, Universidad Politécnica Salesiana.

5. Yu L, Peijian J, Jianming L, Li L, Xuechecng Z, Zhan P, et al. A fast fourier transform-based channel estimation algorithm for MLSE equalization in optical system. In Wireless communications, networking and mobile computing (WICOM); 2011. p. 23-25.

6. Hai-Han L. Performance comparison between DCF and RDF dispersion compensation in fiber optical CATV systems. IEEE Transactions. 2002 Dec; 48(4).

7. Dorini L, Leite N. A general self-dual adaptatitive filtering toggle operator. In Computer graphics and image processing, 2008. SIBGRAPI '08. XXI Brazilian symposium; 2008. p. 189,195,12-15.

8. Mendieta D, Milocco R. Ecualización adaptatica de canales utilizando blanqueo y ajuste de fase. Rev. Arg. de trab. estudiantiles. 2006 Febrero; 1(1).

9. Yi J, Varanasi M, Jian L. Performance analysis of ZF and MMSE equalizers for MIMO systems: An In-Depth study of the high SNR regime. IEEE Transactions. 2011 April; 57(4).

10. Chih-Hao L, Vaidyanathan p. MIMO broadcast DFE tranceivers with QoS contraints: min-power and max-rate solutions. IEEE Transactions. 2013 Noviembre; 61(22).

11. Gorshtein A. Symbol spaced adaptive MIMO equalization for ultrahigh bit rate metro coherent optical links. In IEEE; 2013. p. 414-417.

12. Mori Y. Dual-stage decision-directed phase estimator enabling perfect frequency-offset elimination in digital coherent receivers. In IEEE; 2013. p. 1-3.

13. Li T, Yiguang W, Yuliang G, Lau, Nan C, Chao L. 40 Gb/s CAP32 system with DD-LMS equalizer for short reach óptical transmissions. In IEEE; 2013. p. 2346-2349.

14. Simulación de la interfaz de radio para sistemas de comunicaciones móviles GSM/GPRS. Escuela de ingeniería eléctronica y electrónica, Universidad del Valle.

15. Avalos JF. Algoritmo LMS con error codificado usando un DSP. 2008. Tesis de maestría, Institudo politécnico nacional, Mexico D.F.

16. Granada JJ, Zuluaga CE, Guerrero N, Díaz OM. Compensación de la dispersión cromática usando ecualización LMS. In Symp. de tratamiento de señales, imágenes y visión artificial; 2010; Bogotá. p. 224-229.

17. Poularikas AD, Ramadan ZM. Adaptative Filtering primer with MATLAB. 2006. Taylor \& Francis Group.

18. Roffé E. Uso de los recptores MLSE en las comunicaciones ópticas. 2008. Tesis de pregrado, Universidad central de Venezuela.

19. Binh LN, Huynh TL, Pang KK, Sivahumaran T. MLSE equalizers for frequency discrimination receiver of MSK óptical transmission system. Lightwave technology. 2008 June; 26(12).

20. Shafik RA, Rahman S, Islam R. On the extended relationships among EVM, BER and SNR as performance metrics. In International Conference Electrical and Computer Engineering (ICECE); 2008. p. $408,411,19-21$. 